List your meeting here:

Following is a list of microscopy related meetings and courses. The editors would greatly appreciate input to this list via the electronic submission form under "events/other microscopy events" at the MSA webpage: www.microscopy.org. We will gladly add hypertext links to the notice on the web and insert a listing of the meeting in the next issue. Please furnish the following information (any additional information provided will be edited as required and printed on a space available basis):

- Meeting/Course name

- Meeting/Course dates

- Meeting/Course topic or short description

- Sponsoring society/organization/university

- Contact person

- Telephone number

- Fax number

- Email address

- URL for website

\section{Meetings 2019}

Biophysical Society $63^{\text {rd }}$ Annual

\section{Meeting}

March 2-6, 2019

Baltimore, MD

www.biophysics.org/Meetings/

AnnualMeeting/FutureAnnualMeetings/

tabid/495/Default.aspx

EMIM 2019: $14^{\text {th }}$ European Molecular Imaging Meeting

March 9-22, 2019

Glasgow, UK

www.e-smi.eu/index.php?id=1976

PITTCON Conference \& Expo 2019

March 17-21, 2019

Philadelphia, PA

https://pittcon.org

14th European Molecular Imaging

Meeting - EMIM 2019

March 19-22, 2019

Glasgow, UK

www.e-smi.eu/index.php?id=1976
Frontiers of Characterization and Metrology for Nanoelectronics

March 25-29, 2019

Monterey, CA

www.nist.gov/news-events/events/2017/03/ frontiers-characterization-and-metrologynanoelectro

\section{6th World Conference on Neurology} and Neurosurgery

March 27-28, 2019

Paris, France

www.meetingsint.com/conferences/neurology

\section{ACS Spring Meeting: Chemistry for} New Frontiers

March 31-April 4, 2019

Orlando, FL

www.acs.org/content/acs/en/meetings/ national-meeting/abstract-submission. html?sc=meetings_1800815_mtg_\%20 OR19_od

Symposium "Correlative and in situ microscopy in materials research"

April 1-4, 2019

Regensburg, Germany

regensburg19.dpg-tagungen.de/programm/ abstract

Frontiers of Characterization and Metrology for Nanoelectronics (FCMN)

April 1-4, 2019

Monterey, CA

Contact: Della Miller; e-mail: della@avs.org

Keystone Symposia: Imaging Across Scales - Leveraging the Revolution in Resolution

April 7-10, 2019

Snowbird, UT

www.keystonesymposia.org/19D1

21st International Conference on Microscopy of Semiconducting Materials (MSM-XXI)

April 9-12, 2019

Cambridge, UK

www.events.iop.org/e/21st-internationalconference-on-microscopy-ofsemiconducting-materials-msm-xxi5383d2a4890049da8ad86e47f96f8215/ page.html
Symposium on Recent Advances in Microscopy Characterization of Photonic and Optoelectronic

Materials (POEM2019)

April 10-11, 2019

London, UK

FOM2019: Focus on Microscopy 2019

April 14-17, 2019

London, UK

www.microbeamanalysis.eu/events/ event/57-fom2019-focus-on-

microscopy-2019

Botanical Microscopy 2019

April 14-18, 2019

Oxford, UK

www.rms.org.uk/discover-engage/eventcalendar/botanical-microscopy-2019. html

ICAMME'19 - The International

Conference on Advanced Materials, Microscopy and Energy

April 15-19, 2019

Meknès, Morocco

https://icamme19.sciencesconf.org

MRS Spring Meeting \& Exhibit April 22-26, 2019

Phoenix, AZ

www.mrs.org/spring2019

SVC 62nd Annual Technical

Conference (TechCon'19)

April 27-May 2, 2019

Long Beach, CA

www.svc.org

Sixth International Symposium

Frontiers in Polymer Science

May 5-8, 2019

Budapest, Hungary

www.elsevier.com/events/conferences/ frontiers-in-polymer-science

PICO 2019 - Fifth Conference on

Frontiers of Aberration Corrected

Electron Microscopy

May 5-9, 2019

Kasteel Vaalsbroek, Netherlands

www.er-c.org/pico2019/about.htm 

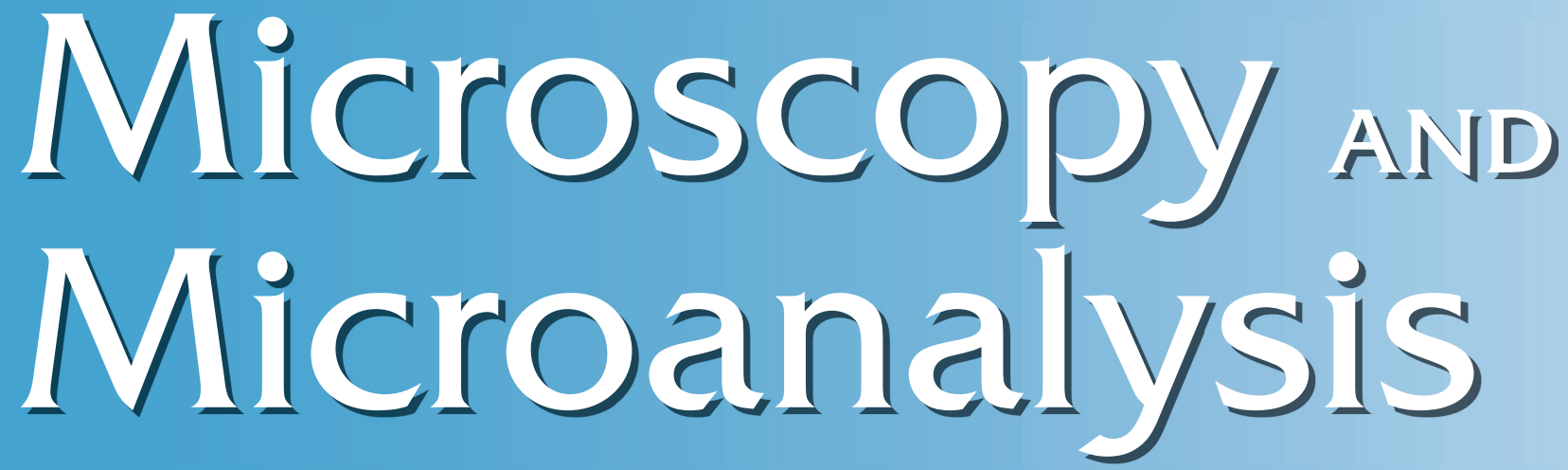

\subsection{4}

2017 Impact Factor

3 out of 10 Microscopy Journals

2017 Journal Citation Reports

(C) Clarivate Analytics

Published for the

Microscopy Society of America

Editor Dr John Mansfield

Microscopy and Microanalysis publishes

original research papers in the fields of

microscopy, imaging, and compositional

analysis. This distinguished international

forum is intended for microscopists in both

biology and materials science.

Online submission at

http://mc.manuscriptcentral.com/mam
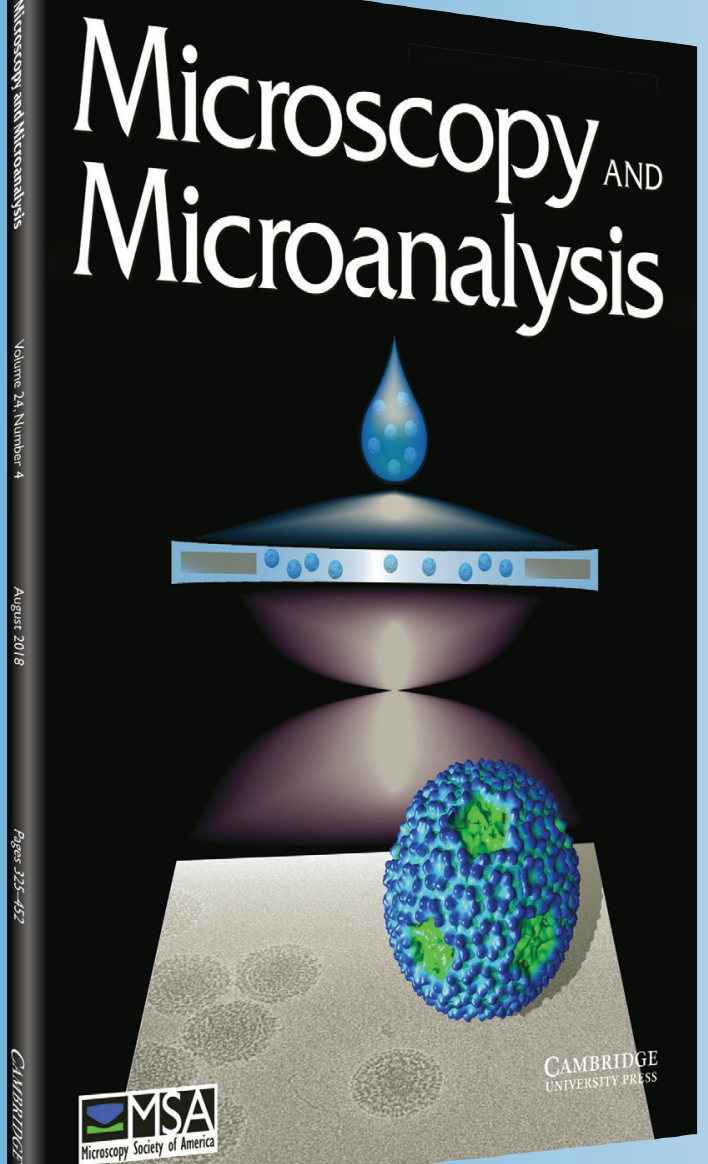

$\infty N$

Microscopy Society of America

cambridge.org/mam 
EMAS 2019 - 16 ${ }^{\text {th }}$ European Workshop on Modern Developments and Applications in Microbeam Analysis

May 19-23, 2019

Trondheim, Norway

https://www.microbeamanalysis.eu

ICMCTF'19- International Conference on Metallurgical Coatings \& Thin Films

May 19-24, 2019

San Diego, CA

www2.avs.org/conferences/ICMCTF

ISPM 2019 - International Scanning

Probe Microscopy Conference

May 26-29, 2019

Louvain-la-Neuve, Belgium

http://uclouvain.be/en/research-institutes/ libst/ispm-2019.html

Advanced Quantitative Transmission

Electron Microscopy: Materials

Research in Several Dimensions

May 27-31, 2019

Nice, France

www.european-mrs.com/advancedquantitative-transmission-electronmicroscopy-materials-research-severaldimensions-emrs

$14^{\text {th }}$ International Symposium on

Macrocyclic and Supramolecular

Chemistry

June 2-6, 2019

Lecce, Italy

https://ismsc2019.eu

Gordon Research Seminar - Three

Dimensional Electron Microscopy

June 8-9, 2019

Hong Kong, China

www.grc.org/three-dimensional-electronmicroscopy-grs-conference/2019

Gordon Research Conference - Three Dimensional Electron Microscopy

June 9-14, 2019

Hong Kong, China

www.grc.org/three-dimensional-electronmicroscopy-conference/2019

$15^{\text {th }}$ International Symposium on Sputtering and Plasma Processes (ISSP2019)

June 11-14, 2019

Kanazawa, Japan

http://issp2019.org

$6^{\text {th }}$ International Symposium on

Advanced Microscopy and

Theoretical Calculations

June 14-15, 2019

Nagoya, Japan

www.amtc6.com

$9^{\text {th }}$ International Colloids Conference June 16-19, 2019

Barcelona, Spain

www.elsevier.com/events/conferences/ international-colloids-conference $6^{\text {th }}$ Nano Today Conference

June 16-20, 2019

Lisbon, Portugal

www.elsevier.com/events/conferences/ nano-today-conference

$4^{\text {th }}$ International Workshop on TEM

Spectroscopy in Material Science

June 17-19, 2019

Uppsala, Sweden

www.teknik.uu.se/applied-materials-

science/research-groups/electron-

microscopy-and-nanoengineering/4th-

international-workshop-on-tem-

spectroscopy-in-material-science/

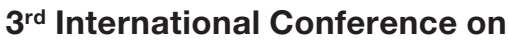

Applied Surface Science

June 17-20, 2019

Pisa, Italy

www.elsevier.com/events/conferences/

international-conference-on-applied-

surface-science

MAS Topical Conference: QMA 2019

June 24-27, 2019

Minneapolis, MN

www.microbeamanalysis.eu/events/event/58-

mas-topical-conference-qma-2019

mmc2019: Microscience Microscopy

Congress 2019

July 1-4, 2019

Manchester, UK

www.mmc-series.org.uk

$1^{\text {th }}$ International Conference on

Nitride Semiconductors

July 7-12, 2019

Bellevue, WA

www.mrs.org/icns-13

Gordon Research Conference -

Tissue Microstructure Imaging

July 7-12, 2019

South Hadley, MA

www.grc.org/tissue-microstructure-

imaging-conference/2019

Edinburgh Super-Resolution Imaging

Consortium (ESRIC) Summer School

July 15-20, 2019

Edinburgh, UK

www.rms.org.uk/discover-engage/eventcalendar/esric-super-resolution-summerschool-2019.html

$1^{\text {th }}$ International Conference on

Atomic Layer Deposition (ALD 2019)

July 21-24, 2019

Bellevue, WA

https://ald2019.avs.org

Microscopy \& Microanalysis 2019

August 4-8, 2019

Portland, OR

Email: nicoleguy@conferencemanagers.com www.microscopy.org
DXC 2019: Denver X-Ray Conference 2019

August 5-9, 2019

Lombard, IL

www.dxcicdd.com

$25^{\text {th }}$ International Congress on X-ray

Optics and Microanalysis (ICXOM-25)

August 5-9, 2019

Lombard, IL

www.dxcicdd.com

MC2019 Microscopy Conference

September 1-5, 2019

Berlin, Germany

www.microscopy-conference.de

\section{EUROMAT 2019}

September 1-5, 2019

Stockholm, Sweden

http://euromat2019.fems.eu

MCM2019: 14th Multinational

Congress on Microscopy

September 15-20, 2019

Belgrade, Serbia

www.sdm.edu.rs/mcm2019

National Society for Histology, $45^{\text {th }}$

Annual Symposium

September 20-25, 2019

New Orleans, LA

http://www.histoconvention.org/futuredates.cfm

$35^{\text {th }}$ North American Molecular

Beam Epitaxy Conference

(NAMBE 2019)

September 25-29, 2019

Boise, ID

E-mail: paulsimmonds@boisestate.edu

Materials Science \& Technology

(MS\&T) 2019

September 29-October 3, 2019

Portland, OR

www.showsbee.com/fairs/42389-Materials-

Science-Technology-2019.html

EMBO|EMBL Symposium: Seeing is

Believing - Imaging the Molecular

Processes of Life

October 9-12, 2019

Heidelberg, Germany

www.embo-embl-symposia.org/

symposia/2019/EES19-09

Neuroscience 2019

October 19-23, 2019

Chicago, IL

Sponsor: Society for Neuroscience

www.sfn.org

AVS $6^{\text {th }}$ International Symposium \&

Exhibition

October 20-25, 2019

Columbus, $\mathrm{OH}$

www.avs.org/symposium 
International Thin Films Conference (TACT2019)

November 17-20, 2019

Taipei, Taiwan

http://tact2019.conf.tw/site/page. aspx?pid=901\&sid=1245\&lang=en

\section{MRS 2019 Fall Meeting}

December 1-6, 2019

Boston, MA

www.mrs.org/fall2019

\section{Courses 2019}

Scanning Electron Microscopy

March 4-8, 2019

Westmont, IL

Sponsor: Hooke College of Applied Sciences www.mccrone.com/scanning-electronmicroscopy-course

Infrared Microscopy

March 18-20, 2019

Westmont, IL

Sponsor: Hooke College of Applied Sciences www.mccrone.com/ftir-training-course

\section{Transgenic Animals -}

Micromanipulation Techniques

March 27-28, 2019

Heidelberg, Germany

Sponsor: EMBL

www.embl.de/training/events/2019/EPP19-01

\section{Atomic Force Microscopy/Scanning}

Probe Microscopy (AFM/SPM)

April 3-4, 2019

Westmont, IL

Sponsor: Hooke College of Applied Sciences www.mccrone.com/afm-training-course

High-Accuracy CLEM: Applications at Room Temperature and in Cryo

April 7-12, 2019

Heidelberg, Germany

Sponsor: EMBL

www.embl.de/training/events/2019/LEM19-01

\section{Gunshot Residue Identification}

May 1-2, 2019

Westmont, IL

Sponsor: Hooke College of Applied Sciences www.mccrone.com/gunshot-residueidentification-course

\section{White-Powder Unknowns}

May 6-10, 2019

Westmont, IL

Sponsor: Hooke College of Applied Sciences www.mccrone.com/white-powderunknowns-course

Fundamentals of Widefield and

Confocal Microscopy and Imaging

May 13-17, 2019

Heidelberg, Germany

Sponsor: EMBL

www.embl.de/training/events/2019/MIC19-01

\section{Sample Preparation Techniques}

May 14-16, 2019

Westmont, IL

Sponsor: Hooke College of Applied

Sciences

www.mccrone.com/sample-preparationtechniques

\section{Advanced Fluorescence Imaging}

Techniques

May 19-24, 2019

Heidelberg, Germany

Sponsor: EMBL

www.embl.de/training/events/2019/MIC19-02

Forensic Hair Analysis

May 20-24, 2019

Westmont, IL

Sponsor: Hooke College of Applied Sciences www.mccrone.com/Forensic-HairAnalysis

Lehigh Microscopy School: Scanning Electron Microscopy, X-ray

Microanalysis, Transmission Electron

Microscopy

June 2-7, 2019

Bethlehem, PA

www.lehigh.edu/microscopy

\section{Polarized Light Microscopy}

June 3-7, 2019

Westmont, IL

Sponsor: Hooke College of Applied Sciences www.mccrone.com/polarized-lightmicroscopy-course

\section{EMAT Workshop on Transmission}

Electron Microscopy

June 11-21, 2019

Antwerp, Belgium

http://ematworkshop.uantwerpen.be

\section{Basic Microscopy}

June 19, 2019

Westmont, IL

Sponsor: Hooke College of Applied Sciences www.mccrone.com/Basic-Microscopy

\section{Super-Resolution Microscopy}

July 8-13, 2019

Heidelberg, Germany

Sponsor: EMBL

www.embl.de/training/events/2019/

MIC19-03

Edinburgh Super-Resolution Imaging

Consortium (ESRIC) Summer School

July 15-20, 2019

Edinburgh, Scotland

www.rms.org.uk/discover-engage/eventcalendar/esric-super-resolution-summerschool-2019.html
EMBL Course: Volume Electron

Microscopy by Automated Serial SEM

October 20-25, 2019

Heidelberg, Germany

www.embl.de/training/events/2019/VEM19-01

Meetings 2020

Biophysical Society $64^{\text {th }}$ Annual Meeting

February 15-19, 2020

San Diego, CA

www.biophysics.org/Meetings/

AnnualMeeting/FutureAnnualMeetings/

tabid/495/Default.aspx

\section{MRS Spring Meeting \& Exhibit}

April 13-17, 2020

Phoenix, AZ

www.mrs.org/spring 2020

ICMCTF'20- International Conference on Metallurgical Coatings \& Thin Films April 26-May 1, 2020

San Diego, CA

www2.avs.org/conferences/ICMCTF/2019/ index.htm

FENS Forum 2020

July 11-15, 2020

Glasgow, Scotland

Email: forum2020@fens.org

www.fens.org/News-Activities/Calendar/

Meetings/2020/07/FENS-Forum-2020

Microscopy \& Microanalysis 2020

August 2-6, 2020

Milwaukee, WI

Email: nicoleguy@conferencemanagers.com www.microscopy.org

emc2020: $17^{\text {th }}$ European Microscopy Congress

August 23-28, 2020

Copenhagen, Denmark

www.emc2020.eu

Neuroscience 2020

October 24-28, 2020

Washington, DC

Sponsor: Society for Neuroscience

www.sfn.org

AVS $67^{\text {th }}$ International Symposium \&

Exhibition

October 25-30, 2020

Denver, CO

www.avs.org/symposium

2020 MRS Fall Meeting \& Exhibit

November 29-December 3, 2020

Boston, MA

www.mrs.org/fall2020 
Meetings 2021

EMAS 2021 - 17th European Workshop on Modern Developments and Applications in Microbeam Analysis

May 16-20, 2021

Krakow, Poland

www.microbeamanalysis.eu/events/ event/60-emas-2021-17th-europeanworkshop-on-modern-developmentsand-applications-in-microbeam-analysis

mmc2021: Microscience Microscopy Congress 2021

July 5-8, 2021

Manchester, UK

www.mmc-series.org.uk

\section{Microscopy \& Microanalysis 2021}

August 1-5, 2021

Pittsburgh, PA

Email: nicoleguy@conferencemanagers.com www.microscopy.org
Microscopy Conference

2021

August 23-27, 2021

Vienna, Austria

www.microscopy-conference.de

Neuroscience 2021

November 13-17, 2021

Chicago, IL

Sponsor: Society for Neuroscience www.sfn.org

\section{Meetings 2022}

\section{FENS Forum 2022}

July 9-13, 2022

Paris, France

Email: forum2022@fens.org

www.fens.org/News-Activities/Calendar/

Meetings/2022/07/FENS-Forum-2022
Microscopy \& Microanalysis 2022

July 31-August 4, 2022

Portland, OR

Email: nicoleguy@conferencemanagers. com

www.microscopy.org

\section{Meetings 2023}

Microscopy \& Microanalysis 2023

July 24-28, 2023

Minneapolis, MN

www.microscopy.org

\section{Meetings 2024}

Microscopy \& Microanalysis 2024

July 28-August 1, 2024

Cleveland, $\mathrm{OH}$

www.microscopy.org

\section{A top journal in Microscopy}

\section{Published for the Microscopy Society of America}

Editor: John Mansfield, University of Michigan, USA

The only journal owned by scientists and published for scientists, Microscopy and Microanalysis provides original research papers in the fields of microscopy, imaging and compositional analysis. This distinguished international forum is intended for microscopists in both biology and materials science.

\section{Online submission at cambridge.org/mam/submit}

View the journal online at cambridge.org/mam

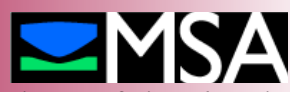

Microscopy Society of America

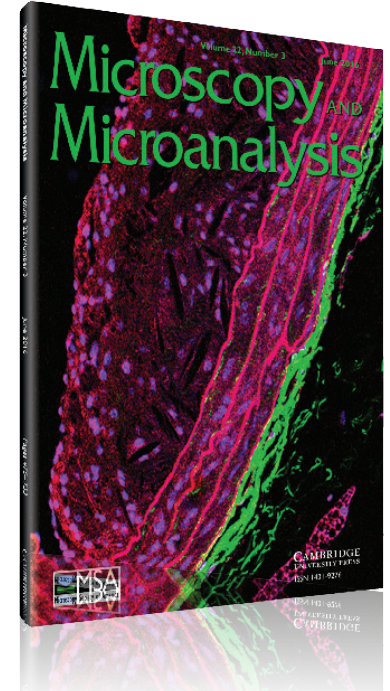

CAMBRIDGE

UNIVERSITY PRESS 